\title{
Mechanical Analysis of Multi-Directional Functionally Graded Cellular Plates
}

\author{
H. Niknam \\ Department of Bioresource Engineering \\ McGill University \\ Montreal, Canada \\ D. Therriault \\ Department of Mechanical Engineering \\ Polytechnique Montreal \\ Montreal, Canada
}

\author{
A. H. Akbarzadeh \\ Department of Bioresource Engineering \& \\ Department of Mechanical Engineering \\ McGill University \\ Montreal, Canada \\ D. Rodrigue \\ Department of Chemical Engineering \\ Université Laval \\ Quebec, Canada
}

\begin{abstract}
In this study, the concept of multi-directional functionally graded cellular material (FGCM) is introduced. FGCMs consist of two spatially-varying engineered phases: solid and void. Different parameters, such as relative density, cell topology, cell orientation, and cell elongation, can be tailored in multiple directions to optimize their mechanical performance. We implement a homogenization technique to evaluate the structural response of plates made by advanced cellular solids. The homogenized effective properties are used in a third-order shear deformation theory (TSDT) formulation. The governing differential equations are solved by a finite element method to predict the mechanical response of FGCM plates. The numerical results reveal that it is possible to increase the buckling load as much as $115 \%$ and decrease the maximum deflection about $60 \%$ by using an FGC structure.
\end{abstract}

Keywords - Architected advanced materials, Functionally graded cellular materials, Homogenization, FDM 3D prining

\section{INTRODUCTION}

Over the last 50 years, several investigations revealed that cellular materials may replace fully dense solids in many different applications [1,2]. Opposed to conventional materials which gain their properties merely from their material composition, cellular solids gain their properties mainly from their underlying architectures [3]. Several investigations have been performed to study the mechanical behavior of cellular materials. The majority of these early investigations on the mechanical properties of advanced porous materials was summarized by Gibson and Ashby in their textbook on "Cellular Solids, Structures and Properties" [1]. Furthermore, application of cellular materials as mechanical elements was first introduced in the design of structural sandwich panels in 1969 when periodic honeycomb sheets were used as the core of sandwich panels [4]. These studies and many others [5-9], shed some light on the properties and possible applications of cellular materials.
The characteristic properties of the representative cell (relative density and void (pore) topology), were uniform across the lightweight structure in all the initial studies mentioned above. However, recent technical developments in advanced manufacturing techniques, like additive manufacturing [10] and powder metallurgy [11], lead to new opportunities to design and manufacture architected cellular structures, in which the geometrical features and material composition of their constituent unit cells can vary in a specified direction. The cellular structures in which the cell' relative density and topology vary across the lightweight structures with a predefined distribution function are called "Functionally Graded Cellular (FGC)" structures. This idea is inspired by natural and biological materials such as bamboo, plants and Humboldt beak.

A few studies are available in the literature implementing the idea of graded cellular properties in the structural mechanical design. One of the earliest research in the field of FGC structures was performed to apply the idea of graded honeycomb structures to obtain a Poisson-curving structure; i.e. a structure which experiences a significant change in its thickness as a result of a prescribed curvature [12]. Afterwards, a finite element based micromechanical model was proposed to predict the fracture toughness of FGC foams [13]. Moreover, FGC structures were shown to be suitable for practical applications such as orthopedic hip implants [14] and cores of aero-engine fan blade [15].

In the present article, we perform a comprehensive study on the architected multi-directional FGCMs with a focus on architected cellular plates. The cell topology is modelled by a superellipse function [16], and the effective properties are obtained by using standard mechanics homogenization [17]. A generalized power-law function is proposed to model the variation of cell characteristics in multiple directions. The governing equations for the structural analysis of FGC plates are obtained by using Reddy's third-order shear deformation theory [18], and solved by a finite element model. The advantages of FGC structures over regular cellular structures are illustrated 
through various examples and the effect of different distribution functions are investigated.

\section{Modelling of FunCtionally GRAded CELLULAR Plates}

\section{A. Formulation of Cell Geometry}

In this work, an FGC plate with length $L_{t o t}$, width $W_{t o t}$, and thickness $T_{\text {tot }}$ is considered in the $x y-$ plane as shown in Fig. 1. The plate consists of $N_{x}$ cells in $x$-direction, $N_{y}$ cells in $y$-direction, and $N_{z}$ cells in $z$-direction, where $(x, y, z)$ refer to the global Cartesian coordinate system also shown in Fig. 1.

The properties of cellular materials depend on their relative density and void topology. Here, a focus is made on extruded two-dimensional square cells in which the void topology of each cell is introduced according to the following superellipse function [16]:

$$
\left[\begin{array}{l}
\tilde{x} \\
\tilde{y}
\end{array}\right]=\left[\begin{array}{l}
r_{x} \cos ^{n} \emptyset \\
r_{y} \sin ^{n} \emptyset
\end{array}\right] R(\theta)
$$

where $0 \leq \emptyset \leq 2 \pi, \tilde{x}$ and $\tilde{y}$ are the local coordinate system whose origin is at the center of the unit cell. Three parameters $\left(r_{x}, r_{y}, n\right)$ have been introduced to control and optimize the void size and topology. Equation (1) simplifies to a circular cell with radius $r_{0}$ for the case of $n=1$ and $r_{x}=r_{y}=r_{0}$. Moreover, $R(\theta)=\left[\begin{array}{cc}\cos \theta & -\sin \theta \\ \sin \theta & \cos \theta\end{array}\right]$ is the rotation matrix enabling to rotate the void topology within each cell. To allow a systematic study for the effect of void topology and void orientation on the mechanical responses of FGC structures, four independent parameters are considered: shape parameter $(n)$, aspect ratio $\left(A . R .=\frac{r_{y}}{r_{x}}\right)$, relative density $\left(\rho_{\text {rel }}\right)$, and orientation angle $(\theta)$. In this study, these four parameters are assumed to vary in the range of $[0.01,3],[1,3],[0.01,1]$ and $[0, \pi]$, respectively. Fig. 2 presents the effect of $n$ and $A$. $R$. on the void topology of periodic cellular materials. It should be mentioned that cell topologies for which the void geometry intersect with cell boundaries has been marked as "Geometrically Inadmissible" in Fig. 2.

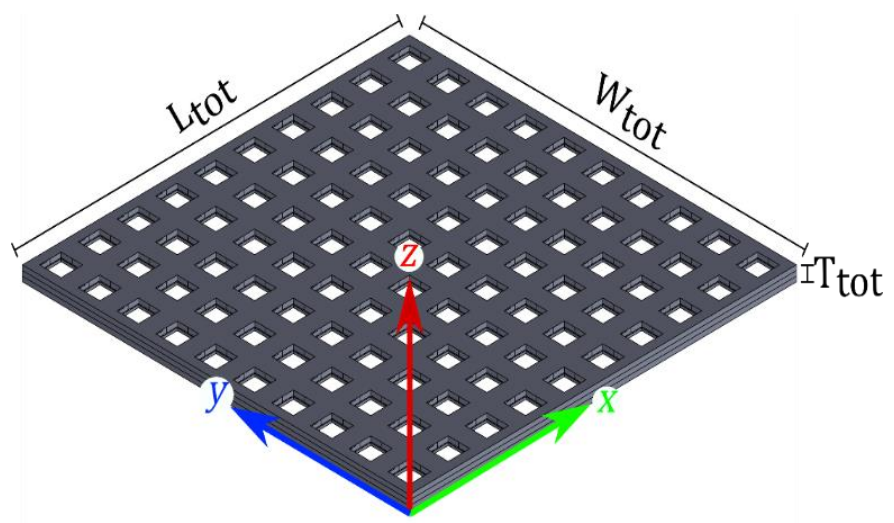

Fig. 1- Homogenous cellular plate

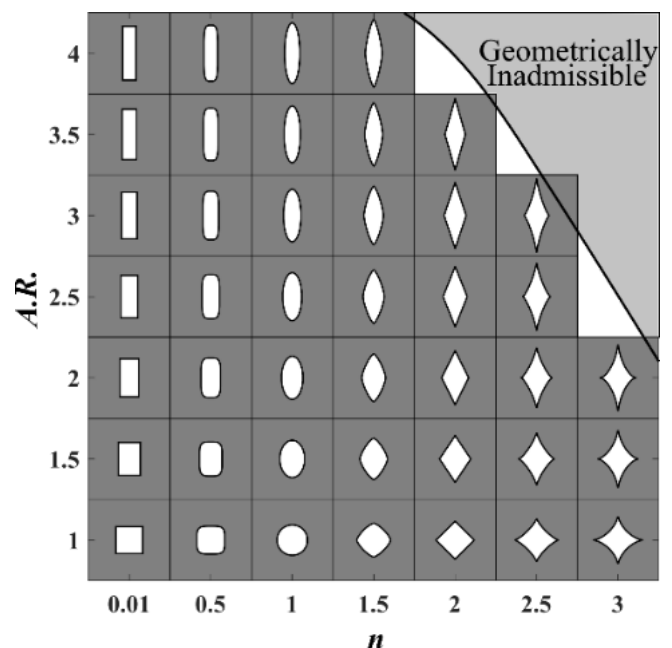

Fig. 2- Achievable cell topologies by a $2 \mathrm{D}$ superellipse function as a function of the shape parameter $(n)$ for $\boldsymbol{\rho}_{\text {rel }}=\mathbf{0 . 9}$ and different aspect ratios (A.R.)

\section{B. Formulation of Cell Topology Variation in FGCMs}

A general power-law function is introduced here to represent any property distribution throughout the length, width and thickness of the material as:

$P(\bar{x}, \bar{y}, \bar{z})=P_{1}+\left(P_{0}-P_{1}\right)\left(1-\frac{\bar{x}}{\bar{x}_{0 P}}\right)^{m x_{P}}\left(1-\frac{\bar{y}}{\bar{y}_{0 P}}\right)^{m y_{P}}\left(1-\frac{\bar{z}}{\bar{z}_{0 P}}\right)^{m z_{P}}$

where $P$ represents any of the four void topology parameters ( $n$, $A . R$., $\rho_{\text {rel }}$, and $\left.\theta\right), P_{0}$ is its value at the origin $(x=0, y=0$, $z=0)$ and $P_{1}$ is its extreme value at $x=\bar{x}_{0 P}, y=\bar{y}_{0 P}$ and $z=$ $\bar{z}_{0 P}$. In all cases, an overbar indicates dimensionless coordinates defined as:

$$
\bar{x}=\frac{x}{L_{\text {tot }}}, \bar{y}=\frac{y}{W_{\text {tot }}}, \bar{z}=\frac{z}{T_{\text {tot }}}
$$

Moreover, $m x_{P}, m y_{P}$ and $m z_{P}$, introduced in Eq. (3), are even integers indicating the distribution profile function. If $m x_{P}$, $m y_{P}$ or $m z_{P}$ equals zero, the property in the $x, y$ or $z$ direction results in a constant value, respectively; i.e. a homogenous distribution of cellular materials in the respective direction. If $P_{0}=P_{1}$, the property has a constant value $P_{0}$ all over the domain.

\section{Formulation of Functionally Graded Cellular Plates}

In this study, the effective elastic properties of all the cells modelled by the superellipse formulation are obtained by standard mechanics homogenization [17]. The material property chart, or so-called Ashby chart [1], is an effective presentation giving an overview of solid materials properties lying in a certain characteristic range. As a result, Fig. 3 presents the Ashby chart for well-known solid materials along with 2D extruder cellular materials with superellipse topologies. Fig. 3 shows that the range of Young's modulus for cellular materials can be extended to regions which cannot be achieved by fully dense solid materials.

To develop a methodology which can be implemented for thin and relativity-thick cellular plates, Reddy's third-order shear deformation theory (TSDT) [18] was used. According to TSDT, the transverse shear stresses are presented as a quadratic 
function through the plate thickness. Therefore, unlike the firstorder shear deformation theory (FSDT), it is not necessary to introduce any shear correction factor in the formulation.

Based on the abovementioned assumptions and by applying Hamilton's approach [18], the governing equations for the present problem, in which the cell topology and subsequently elastic properties are varying in multiple directions, will be obtained as:

$$
\begin{aligned}
& N_{x x, x}+N_{x y, y}=I_{0} \ddot{u}_{0}+J_{1} \ddot{\varphi}_{x}-c_{1} I_{3} \ddot{w}_{0, x} \\
& N_{y y, y}+N_{x y, x}=I_{0} \ddot{v}_{0}+J_{1} \ddot{\varphi}_{y}-c_{1} I_{3} \ddot{w}_{0, y} \\
& c_{1} P_{x x, x x}+2 c_{1} P_{x y, x y}+c_{1} P_{y y, y y}+Q_{x, x}+Q_{y, y}- \\
& 3 c_{1}\left(R_{x, x}+R_{y, y}\right)+q=c_{1}\left(I_{3} \ddot{u}_{0}+J_{4} \ddot{\varphi}_{x}-\right. \\
& \left.c_{1} I_{6} \ddot{w}_{0, x}\right)_{, x}+c_{1}\left(I_{3} \ddot{v}_{0}+J_{4} \ddot{\varphi}_{y}-c_{1} I_{6} \ddot{w}_{0, y}\right)_{, y}+I_{0} \ddot{w}_{0} \\
& M_{x x, x}+M_{x y, y}-c_{1}\left(P_{x x, x}+P_{x y, y}\right)-Q_{x}+3 c_{1} R_{x}= \\
& J_{1} \ddot{u}_{0}+J_{2} \ddot{\varphi}_{x}-c_{1} J_{4}\left(\ddot{\varphi}_{x}+\ddot{w}_{0, x}\right) \\
& M_{y y, y}+M_{x y, x}-c_{1}\left(P_{y y, y}+P_{x y, x}\right)-Q_{y}+3 c_{1} R_{y}= \\
& J_{1} \dot{v}_{0}+J_{2} \ddot{\varphi}_{y}-c_{1} J_{4}\left(\ddot{\varphi}_{y}+\ddot{w}_{0, y}\right)
\end{aligned}
$$

where comma represents partial differentiation with respect to $x$ or $y$, dots denotes differentiation with respect to time and $u_{0}$, $v_{0}, w_{0}, \varphi_{x}$ and $\varphi_{y}$ are the displacements and rotations of the transverse normal on the plane $z=0$. Moreover, $c_{1}=\frac{4}{3 T_{\text {tot }}^{2}}$ and $N_{\alpha \beta}, M_{\alpha \beta}, P_{\alpha \beta}, Q_{\alpha \beta}$ and $R_{\alpha \beta}$ are stress resultants, $I_{i}$ and $J_{i}$ are moments of inertia and $q$ is the transverse mechanical load.

Equations 4-8 are discretized and solved using rectangular conforming element combined with Lagrangian and Hermitian interpolation functions as presented in reference [18].

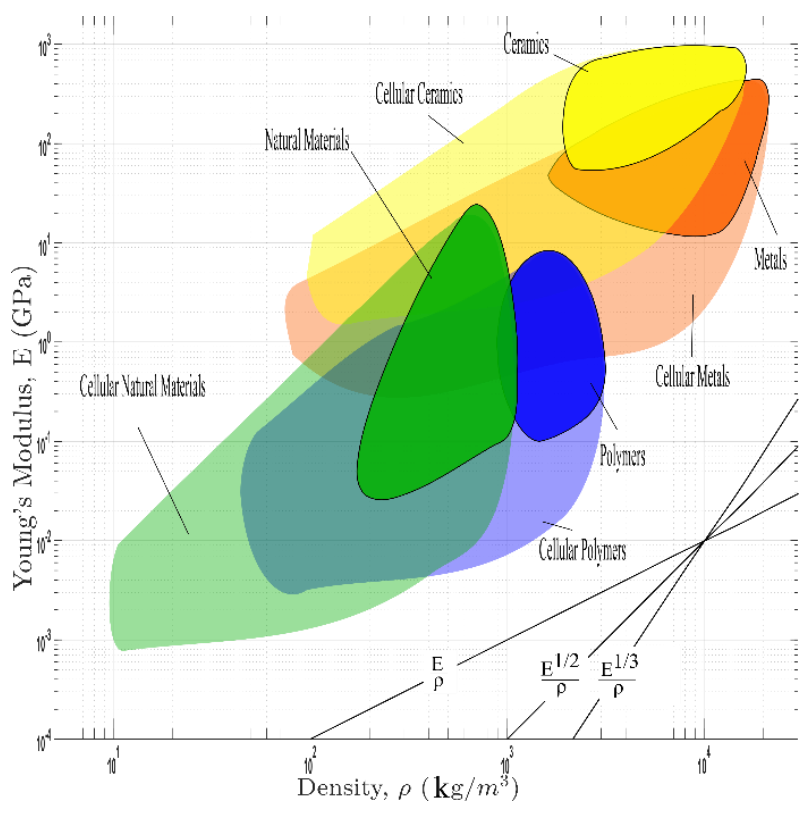

Fig. 3- Ashby chart for cellular materials of superellipse cell topology

\section{RESULTS AND DISCUSSION}

In this section, the numerical results are presented for homogenous and functionally graded cellular materials. The non-dimensional parameters used to represent the mechanical responses of FGC plates are defined as:

$$
\begin{aligned}
& \bar{W}_{\text {max }}=100 W_{\text {max }} \frac{E_{s} W_{\text {tot }}^{3}}{F L_{\text {tot }}^{2}} \\
& \bar{\Omega}=\Omega \frac{L_{\text {tot }}^{2}}{W_{\text {tot }}} \sqrt{\frac{\rho_{s}}{E_{S}}} \\
& \bar{N}_{c r}^{x x}=\frac{10^{4} \widehat{N}_{x x} \lambda L_{\text {tot }}^{2}}{W_{\text {tot }}^{3}}
\end{aligned}
$$

where $F$ is the resultant of the uniform transverse load $(F=$ $q A_{\text {solid }}$ ), while $W_{\text {max }}, \Omega$ and $\widehat{N}_{x x}$ are the maximum deflection of the plate, natural frequency and in-plane compressive load, respectively. Moreover, the size of each constituent unit cell is considered to be the same. Therefore, the total relative density of the cellular plate can be written as:

$$
\rho_{\text {rel,plate }}=\frac{\sum_{i=1}^{N} \rho_{\text {rel }, i}}{N}
$$

where $\rho_{r e l, i}$ is the relative density of $i^{\text {th }}$ cell and $N$ is the total number of cells in a cellular plate.

\section{A. Structural Response of Homogenouos Cellular Plates}

An optimized structural design requires lightweight but stiff structural elements with minimum deflection, maximum mechanical buckling load, and maximum fundamental frequency. The variation of maximum bending deflection as a function of relative density is shown in Fig. 4. The highest stiffness among all the topologies studied in this article is associated with square void. Consequently, it is observed that the maximum deflection of a plate made of square shape voids is the lowest.

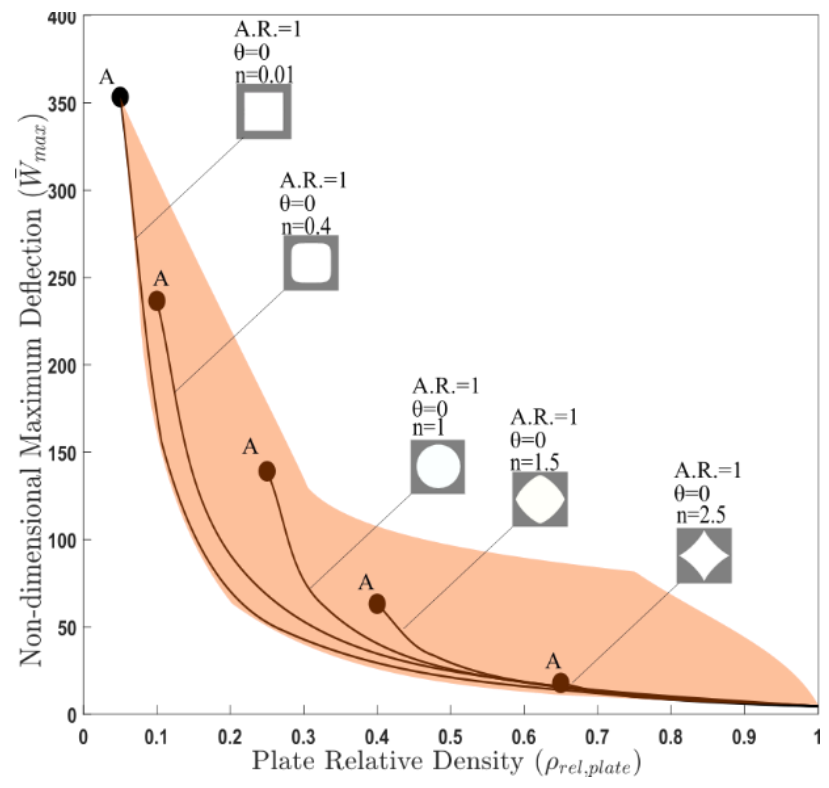

Fig. 4- Non-dimensional maximum deflection of a SSSS cellular plate with $\frac{L_{t o t}}{T_{t o t}}=\mathbf{1 0}$ made of $\mathbf{1 0} \times \mathbf{1 0}$ cells of super ellipsoidal void 


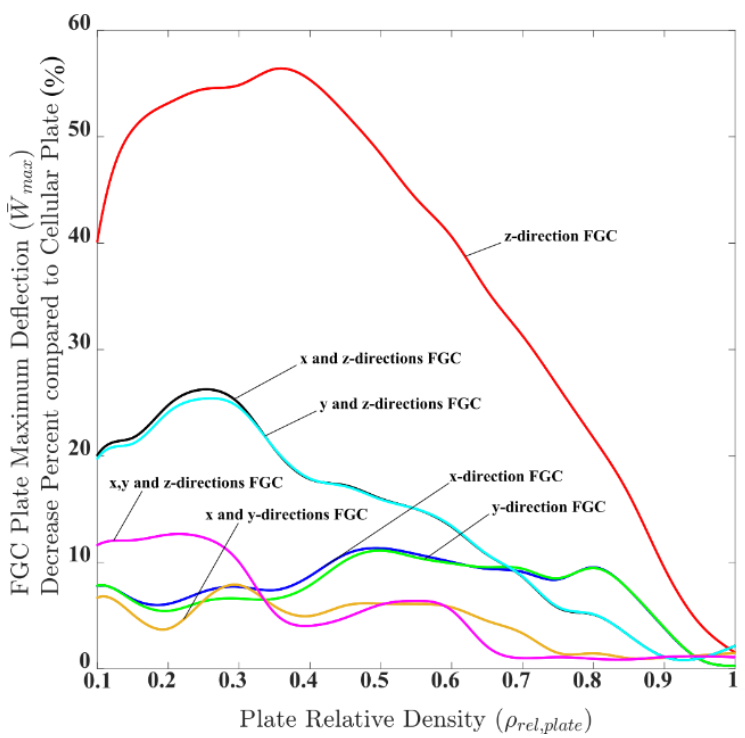

Fig. 5- Decrease of the maximum deflection of alternative multi-directional FGC square plates made by square void shape as a function of the relative density for SSSS plate with $\frac{L_{t o t}}{T_{\text {tot }}}=\mathbf{1 0}$

\section{B. Structureal Response of Multi-Directional FGC Plates}

The effect of density variation in different directions on the stiffening of FGC plates subjected to a uniformly distributed load is presented in Fig. 5. It can be observed that density gradient in the z-direction has the most dominant effect on the maximum deflection variation, while directional density variation in the $x$ - and $z$-directions can decrease the maximum deflection by $26 \%$, the maximum improvement attainable by one directional density variation in the $z$-direction can be up to $56 \%$ for the maximum deflection of architected FGC plates. It is important to note that our conclusion here is only limited to the proposed variation formula defined in Eq. (2) for multidirectional graded cellular materials and for SSSS plates.

To gain insight into the most effective density gradient, the percentage of increase in the critical buckling load is plotted in Fig. 6 as a function of $\left(\rho_{\text {rel } 1}-\rho_{\text {rel0 }}\right)$, where $\rho_{\text {rel } 1}$ is the relative density at $\bar{x}_{0 \rho}=\bar{y}_{0 \rho}=\bar{z}_{0 \rho}=0.5$ and $\rho_{\text {relo }}$ represents the relative density at the origin. Figure 9 shows that for a density gradient in the $z$-direction, the maximum improvement occurs when $\left(\rho_{\text {rel } 1}-\rho_{\text {rel0 }}\right)<0$; i.e. when the center of the FGC plate is more porous than the origin at the FGC plate edges. When the density varies in the $x$-direction or $y$-direction, the conclusion is reversed. Fig. 6 implies that a $z-$ directional FGC plate can have a maximum buckling load when the density at the center of the thickness (mid-plane) is higher at the top and bottom of the plate. For the $x$-directional and $y$-directional FGC plates, higher relative densities at the sides than in the center is more desirable for increasing the critical buckling load. It is also concluded that for all one-directional FGC plates, a higher difference between the side and center density $\left|\rho_{\text {rel1 }}-\rho_{\text {relo }}\right|$, usually leads to a higher critical buckling load increase. It must be noted that the effect of local buckling is not taken into account in the buckling results.

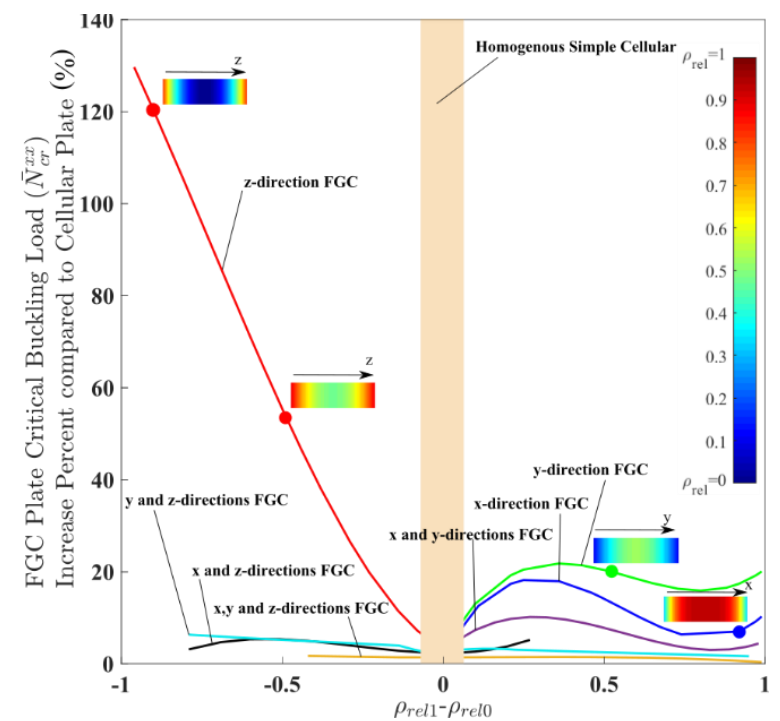

Fig. 6- Effect of the difference between the extreme values of relative density gradient $\left(\boldsymbol{\rho}_{\text {rel1 }}-\boldsymbol{\rho}_{\text {rel2 } 2}\right)$ on the critical buckling load ( $x$-direction) achievable by FGC design for SSSS plate with $\frac{L_{t o t}}{T_{t o t}}=\mathbf{1 0}$

\section{CONCLUSION}

In this work, the concept of multi-directional functionally graded cellular materials was used to produce architected advanced materials. In particular, the mechanical and structural performance of FGCMs were investigated to be used as constitutive elements for lightweight plates. The results showed their important advantages compared to homogenous cellular materials. The superellipse formula was implemented to model the topology of the architected cells and a general power-law function was introduced to control the cell distribution attributes in three different orthogonal directions. The effective cells properties were predicted by standard mechanics homogenization and the functionally graded cellular plate was modeled based on TSDT. The governing equations were solved by using a finite element method. The results clearly showed that architected cellular materials, in the form of superellipsoidal voids, can significantly expand the range of elastic and structural properties leading to lightweight but stiff solutions for advanced materials and structures. Moreover, it was shown that tailoring the topological features of cellular materials through the graded cellular plates thickness more significantly affected their structural response than changing the corresponding features in planar directions.

\section{ACKNOWLEGEMENT}

A.H. Akbarzadeh acknowledges the financial support by McGill University and Natural Sciences and Engineering Research Council of Canada (NSERC) through NSERC Discovery Grant RGPIN-2016-0471. The authors also acknowledge financial support by the Research Center for High Performance Polymer and Composite Systems (CREPEC).

\section{REFERENCES}


[1] Gibson, L.J. and M.F. Ashby, Cellular Solids: Structure and Properties. 1999: Cambridge University Press.

[2] Robert, F., An isotropic three-dimensional structure with Poisson's ratio--1. Journal of Elasticity, 1985. 15: p. 427-430.

[3] A. Akbarzadeh, S. Rankohi, Multifunctional and multiphysics materials as load bearing structural components. 2016.

[4] Allen, H.G., Chapter 4 - Analysis of sandwich beams and struts by strain energy methods, in Analysis and Design of Structural Sandwich Panels. 1969, Pergamon. p. 57-75.

[5] Avalle, M., G. Belingardi, and R. Montanini, Characterization of polymeric structural foams under compressive impact loading by means of energyabsorption diagram. International Journal of Impact Engineering, 2001. 25(5): p. 455-472.

[6] Akbarzadeh, A.H., et al., Dynamic eigenstrain behavior of magnetoelastic functionally graded cellular cylinders. Composite Structures, 2014. 116: p. 404-413.

[7] Andrea, V. and P. Damiano, Analysis and design of lattice materials for large cord and curvature variations in skin panels of morphing wings. Smart Materials and Structures, 2015. 24(3): p. 037006.

[8] Overvelde, J.T.B., S. Shan, and K. Bertoldi, Compaction Through Buckling in 2D Periodic, Soft and Porous Structures: Effect of Pore Shape. Advanced Materials, 2012. 24(17): p. 2337-2342.

[9] Janbaz, S., H. Weinans, and A.A. Zadpoor, Geometrybased control of instability patterns in cellular soft matter. RSC Advances, 2016. 6(24): p. 20431-20436.

[10] Rosen, D.W., Computer-Aided Design for Additive Manufacturing of Cellular Structures. ComputerAided Design and Applications, 2007. 4(5): p. 585594.
[11] Smith, B.H., et al., Steel foam for structures: A review of applications, manufacturing and material properties. Journal of Constructional Steel Research, 2012. 71: p. 1-10.

[12] Lim, T.C., Functionally graded beam for attaining Poisson-curving. Journal of Materials Science Letters, 2002. 21(24): p. 1899-1901.

[13]Lee, S.-J., J. Wang, and B.V. Sankar, A micromechanical model for predicting the fracture toughness of functionally graded foams. International Journal of Solids and Structures, 2007. 44(11): p. 4053-4067.

[14] Arabnejad Khanoki, S. and D. Pasini, Multiscale Design and Multiobjective Optimization of Orthopedic Hip Implants with Functionally Graded Cellular Material. Journal of Biomechanical Engineering, 2012. 134(3): p. 031004-031004-10.

[15] Lira, C., F. Scarpa, and R. Rajasekaran, A Gradient Cellular Core for Aeroengine Fan Blades Based on Auxetic Configurations. Journal of Intelligent Material Systems and Structures, 2011. 22(9): p. 907-917.

[16] Gridgeman, N.T., Lamé ovals. The Mathematical Gazette, 1970: p. 31-37.

[17] Hassani, B. and E. Hinton, A review of homogenization and topology opimization IIanalytical and numerical solution of homogenization equations. Computers \& structures, 1998. 69(6): p. 719-738.

[18]Reddy, J., Analysis of functionally graded plates. International Journal for Numerical Methods in Engineering, 2000. 47(1-3): p. 663-684. 